\title{
Synthetic calibrators for the analysis of total metanephrines in urine: Revisiting the conditions of hydrolysis
}

\author{
Jonathan Simonin ${ }^{\mathrm{a}, 1}$, Sandrine Gerber-Lemaire ${ }^{\mathrm{a}}$, Catherine Centeno ${ }^{\mathrm{b}}$, Caroline Seghezzi ${ }^{\mathrm{b}}$, Katia Iglesias ${ }^{\mathrm{c}}$, \\ Karim Abid ${ }^{\mathrm{b}}$, Eric Grouzmann ${ }^{\mathrm{b}, *}$ \\ a Institute of Chemistry and Chemical Engineering, Ecole Polytechnique Fédérale de Lausanne, Batochime (Bat BCH), CH-1015 Lausanne, Switzerland \\ ${ }^{b}$ Centre Hospitalier Universitaire Vaudois, Division de Pharmacologie et Toxicologie Cliniques, Hôpital Beaumont, Av de Beaumont 29, CH-1011 Lausanne, Switzerland \\ ${ }^{\mathrm{c}}$ Institute of Social and Preventive Medicine (IUMSP), Centre Hospitalier Universitaire Vaudois and University of Lausanne, CH-1011 Lausanne, Switzerland
}

\section{A R T I C L E I N F O}

\section{Article history:}

Received 23 November 2011

Received in revised form 9 February 2012

Accepted 21 February 2012

Available online 3 March 2012

\section{Keywords:}

Metanephrines

Pheochromocytoma

Calibrators

Desulfonation

\begin{abstract}
A B S T R A C T
Background: The quantification of total (free + sulfated) metanephrines in urine is recommended to diagnose pheochromocytoma. Urinary metanephrines include metanephrine itself, normetanephrine and methoxytyramine, mainly in the form of sulfate conjugates (60-80\%). Their determination requires the hydrolysis of the sulfate ester moiety to allow electrochemical oxidation of the phenolic group. Commercially available urine calibrators and controls contain essentially free, unhydrolysable metanephrines which are not representative of native urines. The lack of appropriate calibrators may lead to uncertainty regarding the completion of the hydrolysis of sulfated metanephrines, resulting in incorrect quantification.

Methods: We used chemically synthesized sulfated metanephrines to establish whether the procedure most frequently recommended for commercial kits ( $\mathrm{pH} 1.0$ for 30 min over a boiling water bath) ensures their complete hydrolysis.

Results: We found that sulfated metanephrines differ in their optimum $\mathrm{pH}$ to obtain complete hydrolysis. Highest yields and minimal variance were established for incubation at pH 0.7-0.9 during $20 \mathrm{~min}$.

Conclusion: Urinary pH should be carefully controlled to ensure an efficient and reproducible hydrolysis of sulfated metanephrines. Synthetic sulfated metanephrines represent the optimal material for calibrators and proficiency testing to improve inter-laboratory accuracy.

(c) 2012 Elsevier B.V. All rights reserved.
\end{abstract}

\section{Introduction}

Catecholamines (norepinephrine, epinephrine and dopamine) are important hormones and neurotransmitters involved in cardiovascular and metabolic homeostasis [1]. The physiological effects of catecholamines are terminated by several conjugation pathways including 3-0-methylation followed by sulfonation at the 4-hydroxy group [1]. Norepinephrine and epinephrine are 3-0-methylated by catechol-O-methyltransferase (COMT) into normetanephrine (NMN) (Supplementary data, 2) and metanephrine (MN) (Supplementary data, 1), respectively [1]. Likewise, methoxytyramine (MT) (Supplementary data, $\mathbf{3}$ ) is the O-methylated form of dopamine. Sulfotransferase 1A3 (SULT1A3) catalyzes the transfer of a sulfonyl group from the sulfate donor, 3'-phosphoadenosine-5'-phosphosulfate (PAPS), to the free remaining hydroxy in position 4 of the phenyl ring of metanephrines [2]. SULT1A3 is predominantly expressed in the intestinal mucosa [3].

\footnotetext{
* Corresponding author. Tel.: +412131404 98; fax: +41213147835.

E-mail address: Eric.Grouzmann@chuv.ch (E. Grouzmann).

${ }^{1}$ Current address: Department für Chemie und Biochemie, Universität Bern, Freiestrasse 3, CH-3012 Bern, Switzerland.
}

Pheochromocytoma is a neurendocrinoma that secretes excessive amounts of catecholamines. The diagnosis of this rare disease relies on measures of the concentration of free metanephrines in plasma and total metanephrines in urine and plasma [4]. Sulfated metanephrines predominate over the unconjugated forms in human urine [5], $87 \pm 1 \%, 56 \pm 6 \%$ and $66 \pm 4 \%$ of NMN, MN and MT, respectively, being recovered as sulfoconjugates [6]. Sulfoconjugated metanephrines are highly hydrophilic compounds difficult to directly purify from urine by conventional solid-phase extraction protocol and lack active groups for electrochemical detection. Acidic hydrolysis is therefore, necessary to release the phenolic group for subsequent electrochemical oxidation. LC MS/MS would theoretically allow direct quantification of sulfated metanephrines without prior hydrolysis but no efficient solid-phase extraction method has been yet validated for conjugated and unconjugated metanephrines.

Total urinary metanephrines are usually measured after an acid hydrolysis step or more rarely by enzyme treatment with arylsulfatase to cleave the sulfate moiety from the phenolic group [7-17].

Metanephrines in free form are measured by HPLC with electrochemical or fluorimetric detection, using calibrators prepared inhouse by weighting free synthetic metanephrines or using certified 
calibrators provided by commercial distributors such as BioRad, Chromsystems or RECIPE [7-17]. It has been reported that most metanephrines present in calibrators and quality controls are free rather than sulfoconjugated forms [18]. Therefore, commercial and in-house calibrators based on free forms are inadequate to standardize sulfated metanephrine hydrolysis because they suffer from two potential bias: 1) deconjugation yield cannot be assessed and 2) urine samples used as calibrators may contain small amounts of sulfoconjugated metanephrines which upon hydrolysis will increase the value of free, spiked metanephrines measured, resulting in an over-estimation of the calibrator values. Even though an internal standard is used to assess the yield of the solid-phase extraction procedure for free metanephrines, it does not guarantee full hydrolysis of sulfated metanephrines.

The gold standard conditions for desulfonation of metanephrines, initially established by Pisano in 1960, consisted of acidic treatment at $\mathrm{pH} 0.5-0.9$ for $20 \mathrm{~min}$ [16]. Further adaptations led to routine procedures in clinical laboratories where acidic treatment at $\mathrm{pH}$ values ranging from 0.5 to 1.1 and incubation time from 20 to 45 min are commonly applied [7-17]. The problem is that, should shortcomings occur at this step (e.g., insufficient acidification), or should this step be omitted, the commercially available quality control samples will fail to alert the analyst. Assay batches will pass quality control even though concentrations of the clinical specimens will be severely underestimated, leading to false-negative results with a real potential for missed diagnosis of dangerous tumors.

The aim of the present work was to synthesize sulfoconjugated metanephrines and optimize their use (Supplementary data, 4-6) as calibrators and more importantly as quality control in the measurement of fractionated urinary metanephrines.

\section{Material and methods}

\subsection{Reagents, materials and instruments}

\subsubsection{Reagents and materials}

All commercially available reagents and solvents (Fluka/Aldrich, Buchs, $\mathrm{CH}$ ) and Acros (Wohlen, $\mathrm{CH}$ ) were used without further purification. NMN and MT were purchased from Sigma-Aldrich (St. Louis, Mo, USA) and MN was supplied by Isosciences (King of Prussia, PA, USA). For reactions requiring anhydrous conditions, dry solvents were obtained by filtration (Innovation Technology). In the absence of specific instructions, the experiments were carried out under argon atmosphere. Reactions were monitored by thin-layer chromatography (Merck silica gel $60 \mathrm{~F}_{254}$ plates). Detection was by UV light, or using $\mathrm{KMnO}_{4}$ or Pancaldi reagents $\left[\left(\mathrm{NH}_{4}\right)_{6} \mathrm{MoO}_{4}, \mathrm{Ce}\left(\mathrm{SO}_{4}\right), \mathrm{H}_{2} \mathrm{SO}_{4}\right.$, $\left.\mathrm{H}_{2} \mathrm{O}\right]$. Purifications were performed by flash chromatography on silica gel (Merck, Zoug, CH) N 9385 silica gel 60, 240-400 mesh and reverse phase HPLC.

\subsubsection{Instruments}

${ }^{1} \mathrm{H}-\mathrm{NMR}$ spectra: Bruker ARX-400, Bruker DPX-400 spectrometers at $400 \mathrm{MHz}$ and Bruker AVII-800 spectrometers at $800 \mathrm{MHz}$. Chemical shifts in ppm relative to the solvent's residual ${ }^{1} \mathrm{H}$ signal (MeOD: $3.34 \mathrm{ppm}, \mathrm{CDCl}_{3}: 7.27 \mathrm{ppm}, \mathrm{C}_{6} \mathrm{D}_{6}: 7.30 \mathrm{ppm}$ ) as internal reference. ${ }^{1} \mathrm{H}$ assignments were confirmed by 2D-COSY spectra. Multiplicity reflects apparent patterns. Coupling constants $J$ in $\mathrm{Hz}$; b stands for broad. ${ }^{13} \mathrm{C}$-NMR spectra: same instrument as above at $101 \mathrm{MHz}$. Reference for solvents used as internal reference in ppm (MeOD: $49 \mathrm{ppm}$, $\mathrm{CDCl}_{3}$ : $77 \mathrm{ppm}, \mathrm{C}_{6} \mathrm{D}_{6}: 128.5 \mathrm{ppm}$ ). Coupling constants $\mathrm{J}$ in $\mathrm{Hz} ;{ }^{13} \mathrm{C}$ assignments were confirmed by 2D-HSQC spectra. IR spectra: Perkin Elmer Paragon 1000 FT-IR spectrometer. Mass spectra: MALDI-TOF spectrometer (Axima-CFR ${ }^{+}$, Kratos, Manchester, UK); ESI-Q spectrometer (Finnigan SSQ 710 C, Thermoquest, UK); HRMS-ESI spectrometer (Q-Tof Ultima spectrometer, Micromass, Manchester, UK). The high purity of the synthetic sulfoconjugated metanephrines (>99\%) was assessed by ${ }^{1} \mathrm{H}$ and ${ }^{13} \mathrm{NMR}$ spectra and HRMS-ESI analysis. Elemental analysis was not possible due to the high hygroscopicity of the products.

\subsection{Synthesis and characterization of sulfated metanephrines}

The chemical protocols used for the synthesis of each sulfated metanephrine (Supplementary data compounds 4, $\mathbf{5}$ and $\mathbf{6}$ ) are available in the supporting information section.

\subsection{Quantification of sulfated metanephrines}

Sulfated metanephrines were quantified by two independent assays; 1 . LC-UV quantification: total disappearance of the sulfated compounds upon hydrolysis $\left(100{ }^{\circ} \mathrm{C}\right.$ at $\mathrm{pH} 1.0$ for $30 \mathrm{~min}$ ) and concomitant appearance of newly formed free metanephrines were observed and quantified by LC-UV using solutions of free metanephrines prepared in house and confirmed by the value assigned with the BioRad calibrator (Reinach, Switzerland) for metanephrines (see below). 2) LC-MS/MS quantification: solutions of sulfated metanephrines were also directly quantified by a newly developed LC-MS/MS method (unpublished data). The concentrations found for the three sulfated metanephrines using these two independent analytical methods were highly consistent since percentage between assigned and measured values measured by LC-MS/MS and LC-UV, respectively, and challenged with BioRad calibrators were $109 \%$ and $107 \%$ for sulfated normetanephrine (S-NMN), $109 \%$ and $105 \%$ for sulfated metanephrine (S-MN) and $105 \%$ and $86 \%$ for sulfated methoxytyramine (S-MT). Regular certified calibrator solutions were BioRad Urine Standard (catalog nos. 195-5846) batch 1809 containing: NMN: 3925 nmol/l, MN: 1795 nmol/l, MT: 1878 nmol/l. Internal quality controls were a kind gift from RECIPE (Munich, Germany) (catalog nos. 8822). Urine calibrator and control lyophylisates were reconstituted accordingly to the instructions of the supplier. These two methods of quantification enabled us to titer our stock solution at: $3730 \mu \mathrm{mol} / \mathrm{l}$ for S-NMN, $400 \mu \mathrm{mol} / \mathrm{l}$ for S-NMN and $3800 \mu \mathrm{mol} / \mathrm{l}$ for S-MT.

\subsection{Validation of hydrolysis conditions}

The biological matrix used included charcoal-stripped human urine (Golden West Biologicals, Temecula, USA), urine calibrator from Biorad, control level 1 from RECIPE, one urine spot collected from a pool of 40 volunteers and one urine spot from one volunteer (Vol01). Each matrix was tested for free metanephrines content before and after hydrolysis, with spiked urines containing the following amounts of sulfated metanephrines: Spike 1: S-NMN (2800 nmol/l), S-MN (1400 nmol/l) and S-MT (1800 nmol/l); Spike 2: S-NMN (1400 nmol/l), S-MN (700 nmol/l) and S-MT (900 nmol/l); Spike 3: S-NMN (700 nmol/l), S-MN (350 nmol/l) and S-MT (450 nmol/l). Free metanephrines were measured as indicated by the protocol provided by RECIPE. Spiked amount of S-MNs was chosen to mimick normal and pathological concentrations found during routine measurements. The initial $\mathrm{pH}$ of urine (charcoal stripped human urine, Biorad calibrator, RECIPE internal quality controls and urine pool) was adjusted at $\mathrm{pH} 1.0$ and stored at $-20^{\circ} \mathrm{C}$. The internal standard 3-methoxy-4-hydroxybenzylamine (MHBA) was added prior to hydrolysis and $\mathrm{pH}$-adjusted with $\mathrm{HCl}$ or $\mathrm{NaOH}$ to reach a given $\mathrm{pH}$ that ranged from 0.5 to 7.0 (see below). Hydrolysis was carried out over a boiling bath $\left(100^{\circ} \mathrm{C}\right)$ during a period ranging from 1 to 60 min (see below). Free metanephrines were then measured using the RECIPE metanephrines extraction kit (RECIPE, catalog nos. 4000). Forty microliters of eluate was injected into an isocratic HPLC model equipped with a dual piston pump and an autoinjector (model 542) coupled to a 5600A electrochemical Coularray system (ESA-Dionex, Sunnyvale, CA USA). The oven temperature for the column 
gasket was set to $30^{\circ} \mathrm{C}$ and flow rate adjusted to $1 \mathrm{ml} \mathrm{min}^{-1}$. The detection was done with one cell module containing four electrochemical detector cells with cell potentials maintained at 240,320, 340 and $360 \mathrm{mV}$, beginning with the first serially aligned sensor [4]. The inter-assay quality control was assessed by $\mathrm{C} 1$ value determination from RECIPE; coefficient of variation was $2.0 \%$ for normetanephrine ( $1400 \mathrm{nmol} / \mathrm{l}$ ), $4.0 \%$ for metanephrine $(715 \mathrm{nmol} / \mathrm{l})$ and $4.0 \%$ for methoxytyramine $(900 \mathrm{nmol} / \mathrm{l})$.

\subsection{1. $\mathrm{pH}$ range and hydrolysis yields}

Charcoal stripped urine and native urine samples (Vol01) were both supplemented with spike 2 and heated $\left(100{ }^{\circ} \mathrm{C}\right.$ for $\left.30 \mathrm{~min}\right)$ at various $\mathrm{pH}(0.5,1.0,1.5,2.0$ and 3.0). Free metanephrine concentrations obtained after hydrolysis, were compared with those produced by hydrolysis at $\mathrm{pH} 1.0$ for $30 \mathrm{~min}$ (gold standard, considered as quantitative hydrolysis). Controls consisted of Biorad calibrator and unspiked native urine.

\subsubsection{Time range and hydrolysis yield}

Incubation times $(0,1 \mathrm{~min}, 5 \mathrm{~min}, 10 \mathrm{~min}, 20 \mathrm{~min}, 30 \mathrm{~min}$ and $60 \mathrm{~min}$ ) were chosen for the hydrolysis of sulfated metanephrines in a native urine pool collected from several volunteers and supplemented with spike 2 . The experiments were performed at $\mathrm{pH} 1.0$ to meet the recommended procedures.

\subsubsection{Simultaneous effect of $\mathrm{pH}$ and duration on the rate of hydrolysis}

Samples of the urine pool were adjusted to five $\mathrm{pH}$ values by 0.2 unit increments starting from $\mathrm{pH} 0.5$ up to 1.5 . For each $\mathrm{pH}$ value, hydrolysis was performed for $20,25,30$ and $35 \mathrm{~min}$ at $100^{\circ} \mathrm{C}$. Half of the samples were spiked with sulfated metanephrines (spike 2). A total of 456 measurements were performed within 23 experiments during 3 months by two experienced technicians to take day-to-day variability into account.

\subsection{Statistical analyses}

In order to compare the mean concentrations of NMN, MN and MT obtained under different conditions (calibrator, calibrator and addition of exogenous synthetic sulfated metanephrines at three levels of concentration), non-parametric tests (Kruskal-Wallis equality-ofpopulations rank test) were run for each metanephrine. For significant differences, Mann-Whitney post tests were also carried out to compare calibrator means after each addition of exogenous synthetic sulfated metanephrines and Bonferroni corrections were used to take multiple testing into account (alpha of $1.667 \%$ instead of $5 \%$ ).

To evaluate the optimal conditions for hydrolysis of sulfated metanephrines (greatest mean concentration and the lowest variability of measurements), linear multiple regressions were calculated for MN, NMN and MT with time $(20,25,30$ and 35 min taking 30 min as reference group) and $\mathrm{pH}(0.5-0.7,0.7-0.9,0.9-1.1,1.1-1.3$ and $1.3-1.5$ with the interval of $0.9-1.1$ as reference group) as covariables. These models were adjusted for spike effects by introducing a spike factor (spike vs no spike, no spike as reference group) and its interaction with $\mathrm{pH}$. The outcome was the amount of sulfated metanephrines in urine. Therefore for the spiked urines, this quantity was defined as the total sulfated metanephrines found minus the theoretical spike added to the urine. As there was a spike effect (main effect of spike with an underestimation of the three metanephrines and an interaction between spike and $\mathrm{pH}$ for the S-NMN and the S-MN), the spiked and unspiked urines were analyzed separately. Second, Levene's tests of homogeneity of variance were run to evaluate the optimal conditions to minimize variability around each mean. Desulfonation was assumed to have taken place from the point in time when only values below the confidence limits were observed. Desulfonation was considered relevant when it exceeded $10 \%$.

\section{Results}

\subsection{Synthesis of sulfated metanephrines}

While Hegedus reported the preparation of sulfoconjugates $\mathbf{5}$ and $\mathbf{6}$ using chlorosulfonic acid and pyridine, a general protocol for the efficient and selective sulfoconjugation of metanephrines and analogs was not available [19]. Starting from free metanephrines, we aimed at sulfonation of the phenol moieties under mild conditions. To this end, adequate protection of the other reactive functions was required. 3-Methoxytyramine (3) was transformed into the corresponding tert-butyl carbamate in almost quantitative yield (Supplementary data).

All attempts to obtain direct sulfonation of the phenolic group with $\mathrm{SO}_{3}$-amine complexes [20-24] were unsuccessful leading to decomposition of the starting material or to complex mixtures. An alternative strategy based on the use of the protected sulfonation reagent $\mathbf{8}[25,26]$ was investigated $[27,28]$. Following the procedure of Taylor [29], treatment of phenol 7 (0.3 M concentration in the reaction medium) with an excess of $\mathbf{8}$ in the presence of triethylamine and DMAP afforded the sulfate diester $\mathbf{1 0}$ in $94 \%$ yield. Removal of the carbamate moiety by trifluoroacetic acid in water led to a concomitant complete desulfonation with recovery of the starting 3-methoxytyramine (3). Gratifyingly, a sequence of hydrogenolysis catalyzed by Pd/C using ammonium formate as source of hydrogen [30], followed by cleavage of the tert-butyl carbamate in the presence of $5 \%$ trifluoroacetic acid in dichloromethane, provided the desired aryl sulfate $\mathbf{6}$ in 95\% yield (2 steps).

The sulfonation of normetanephrine and metanephrine followed a similar approach (Supplementary data). After protection of the amines as the tert-butyl carbamates $\mathbf{1 1}$ and $\mathbf{1 8}$ in high yield, a first attempt of sulfonation of $\mathbf{1 1}$ with reagent $\mathbf{8}$ resulted in a low conversion toward a mixture of the desired sulfate diester $\mathbf{1 2}$ (11\% yield) and the product of sulfonation on the side-chain hydroxyl group (13, 17\% yield). Intermediates $\mathbf{1 1}$ and $\mathbf{1 8}$ were thus converted into the bis-tertbutyldimethylsilyl ethers. According to the methodology developed by Jiang et al. [30], the phenol moiety was selectively deprotected in the presence of cesium carbonate to afford phenols 15 and 19 in high yields. Further sulfonation with excess of $\mathbf{8}$ delivered the expected diesters $\mathbf{1 9}$ and $\mathbf{2 0}$ in 88 and 75\% yield, respectively. After fluoride-promoted removal of the silyl protecting group, hydrogenolysis in the presence of $\mathrm{Pd} / \mathrm{C}$ and ammonium formate was not met with success. Removal of the trichloroethyl group was thus carried out with zinc and ammonium formate [29]. Final cleavage of the carbamate moieties delivered the sulfoconjugated normetanephrine $\mathbf{5}$ and sulfoconjugated metanephrine $\mathbf{4}$ in good yield.

The use of chlorosulfuric acid 2,2,2-trichloroethyl ester provided an efficient alternative for the sulfonation of metanephrines and analogs which could not be achieved with classical methodologies using $\mathrm{SO}_{3}$-amine complexes. With the protocols disclosed herein, sulfoconjugate $\mathbf{6}$ was obtained in 4 steps and 79\% overall yield from the parent 3-methoxytyramine while sulfoconjugated normetanephrine and metanephrine were delivered in 7 steps with 35 and 25\% overall yields respectively.

\subsection{Sulfated metanephrines in urine calibrator and control}

The Biorad calibrator and the control level 1 (C1) from RECIPE contain low amounts of sulfated metanephrines $(<2 \%$ for S-NMN; $<7 \%$ for S-MN; $<6 \%$ for S-MT) since the concentration of metanephrines is not significantly different before and after hydrolysis for calibrator and C1 without spikes (A vs B and C vs D, respectively, Supplementary data). The addition of exogenous synthetic sulfated metanephrines at three concentration levels of concentration in the calibrator and $\mathrm{C}$, while it was not detected in unhydrolyzed urines is clearly measured after hydrolysis (ratio of found versus expected 
concentrations ranging from 95.8 to $106.9 \%$ for S-NMN, 92.8 to $100.9 \%$ for S-MN and 100.3 to $108.2 \%$ for S-MT, B and D, Supplementary data).

\subsection{Behavior of synthetic and endogenous sulfated metanephrines toward hydrolysis under different $\mathrm{pH}$}

Optimal hydrolysis was observed when samples were adjusted at $\mathrm{pH} 1.0$ prior to heating at $100{ }^{\circ} \mathrm{C}$ for $30 \mathrm{~min}$. Increasing the $\mathrm{pH}$ led to a dramatic decrease of hydrolysis yield since at pH 2.0 only $25 \%$ of SNMN, $45 \%$ of S-MN and 18\% of S-MT were hydrolyzed. No hydrolysis was observed for $\mathrm{pH}$ values above 2.0 . Besides, heating at $100{ }^{\circ} \mathrm{C}$ is a prerequisite to obtain desulfonation since incubation at $\mathrm{pH} 1.0$ for

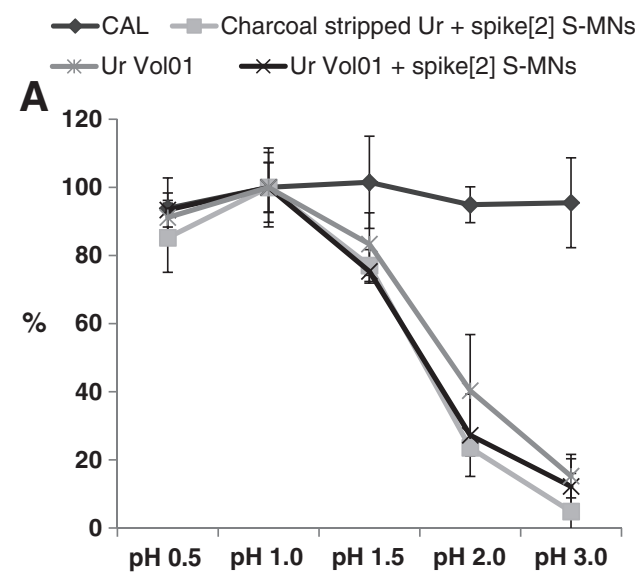

B

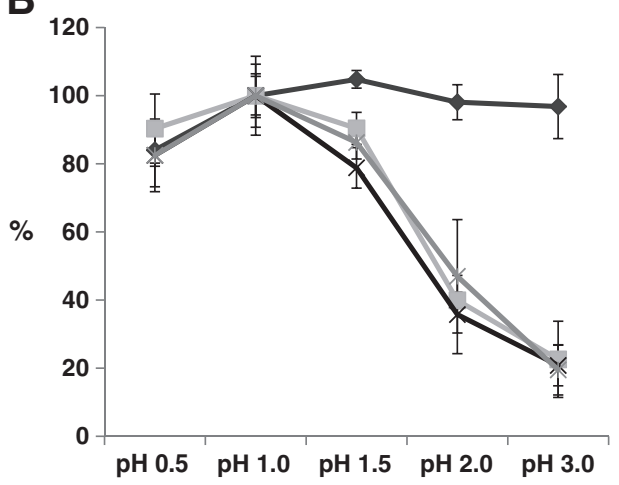

C

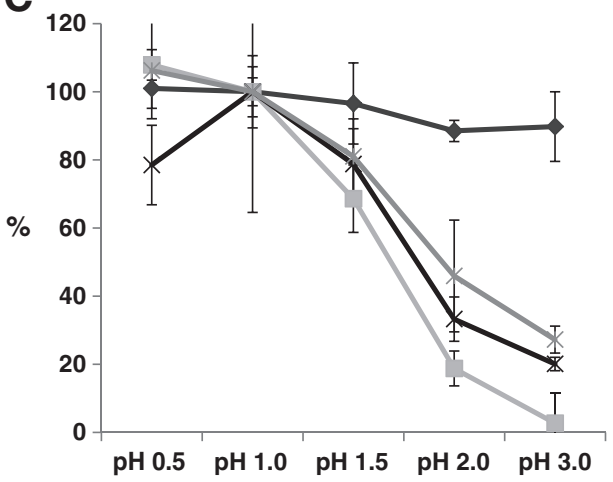

Fig. 1. Quantification of NMN (A), MN (B) and MT (C) in charcoal-stripped urine complemented with S-MNs from spike 2, urine from one volunteer (Vol01) with S-MNs from spike 2 and the same urine not complemented with S-MNs. Samples were adjusted to $\mathrm{pH}$ values ranging from 0.5 to 3.0 and heated. Values were expressed in \%, compared to values obtained for corresponding samples at $\mathrm{pH} 1.0(\mathrm{pH}$ of reference according to the guidelines). Biorad Calibrator was used as control.
30 min at $20^{\circ} \mathrm{C}$ did not affect the stability of sulfated metanephrines (data not shown).

Since the optimal pH range for hydrolysis appeared narrow, we evaluated whether the hydrolysis of synthetic and endogenous sulfated metanephrines was similar between $\mathrm{pH} 0.5$ and 3.0. We observed that hydrolysis yields for the three sulfated metanephrines were similar in the spiked charcoal stripped urine (spike 2), the native urine and the spiked native urine (Fig. 1A for S-NMN, B for S-MN, C for S-MT). However, considering hydrolysis conditions at $\mathrm{pH} 1.0$ for $30 \mathrm{~min}$ as the gold standard, it appears that $\mathrm{pH}$ tolerance around this target is limited since hydrolysis of sulfated metanephrines was already reduced by approx. $20 \%$ at $\mathrm{pH} 0.5$ and 1.5 .

\subsection{Effect of incubation time on hydrolysis yield}

Hydrolysis of the three sulfated metanephrines at $100{ }^{\circ} \mathrm{C}$ started after $1 \mathrm{~min}$ of incubation to reach $60 \%$ of desulfonation after $5 \mathrm{~min}$. A plateau was reached between 20 AND 30 min for both native (Fig. 2A) and spiked (Fig. 2B) urines.

\subsection{Simultaneous effects of $\mathrm{pH}$ and duration on hydrolysis yields}

We conducted similar experiments as those described above (incubation time) using a much larger set of samples (456 samples in 23 experiments during 3 months) in order to test reproducibility and increase the accuracy of the results including $\mathrm{pH}$ variability within short intervals (0.5 to 1.5). We found no significant time effect between 20 and $35 \mathrm{~min}$ on the yield of desulfonation for the three
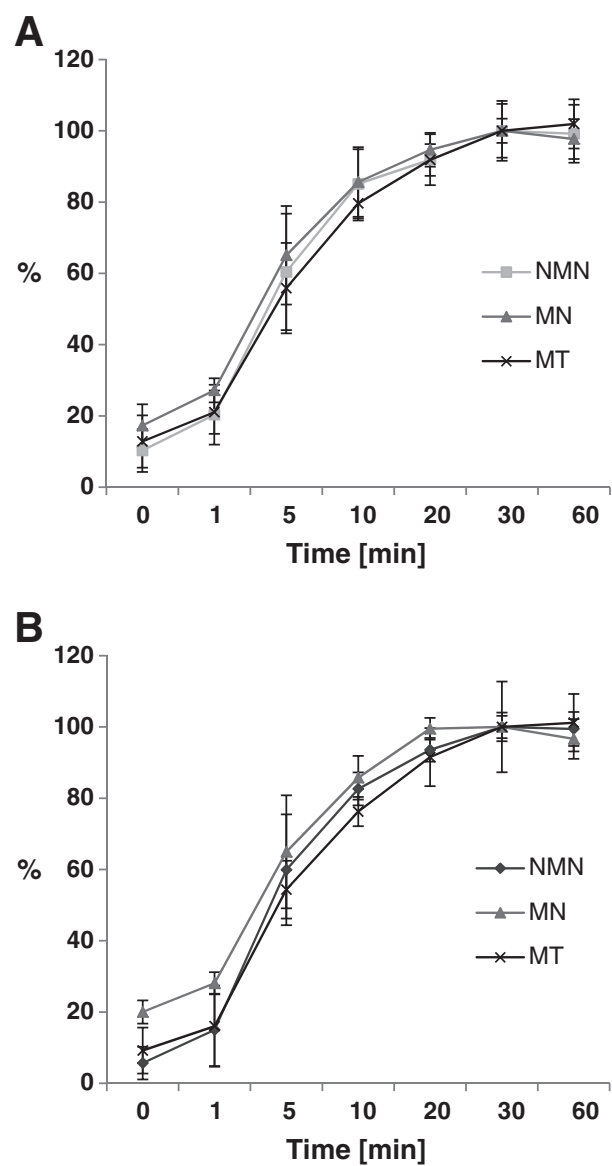

Fig. 2. (A) Quantification of $N M N, M N$ and $M T$ in pooled urine from 40 volunteers adjusted to $\mathrm{pH} 1.0$ prior to incubation at $100{ }^{\circ} \mathrm{C}$ at various time points ( 1 to $60 \mathrm{~min}$ ). Values were expressed in \%, compared to values obtained for corresponding samples incubated for $30 \mathrm{~min}$ (time of reference according to the guidelines). (B) The urine pool was spiked with S-MNs originating from spike 2. 
Table 1

Simultaneous effects of $\mathrm{pH}$ and duration on the percentage of hydrolysis of sulfated metanephrines for unspiked urines.

\begin{tabular}{|c|c|c|c|c|c|c|c|c|c|}
\hline & \multicolumn{3}{|l|}{ NMN } & \multicolumn{3}{|l|}{ MN } & \multicolumn{3}{|l|}{ MT } \\
\hline & Beta & Confidence interval at $95 \%$ & p-Value & Beta & Confidence interval at 95\% & p-Value & Beta & Confidence interval at $95 \%$ & p-Value \\
\hline \multicolumn{10}{|c|}{ Time reference: 30 min } \\
\hline $20 \mathrm{~min}$ & -17.67 & {$[-61.08 ; 25.73]$} & 0.423 & -5.41 & {$[-25.01 ; 14.2]$} & 0.587 & -59.95 & {$[-196.59 ; 76.69]$} & 0.388 \\
\hline $25 \min$ & -7.36 & {$[-50.28 ; 35.55]$} & 0.736 & 0.34 & {$[-19.05 ; 19.72]$} & 0.973 & -31.78 & {$[-166.88 ; 103.33]$} & 0.643 \\
\hline $35 \mathrm{~min}$ & 5.04 & {$[-37.71 ; 47.78]$} & 0.817 & 4.24 & {$[-15.07 ; 23.54]$} & 0.666 & 21.32 & {$[-113.24 ; 155.88]$} & 0.755 \\
\hline \multicolumn{10}{|c|}{ pH reference: [0.9-1.1] } \\
\hline$[0.5-0.7]$ & -60.20 & {$[-107.84 ;-12.56]$} & 0.014 & -50.95 & {$[-72.47 ;-29.43]$} & 0.000 & -62.36 & {$[-212.34 ; 87.62]$} & 0.413 \\
\hline$[0.7-0.9]$ & 35.03 & {$[-6.17 ; 76.24]$} & 0.095 & -2.69 & {$[-21.3 ; 15.92]$} & 0.776 & 170.57 & {$[40.86 ; 300.28]$} & 0.010 \\
\hline$[1.1-1.3]$ & 7.70 & {$[-39.93 ; 55.32]$} & 0.750 & 10.58 & {$[-10.93 ; 32.09]$} & 0.333 & -46.78 & {$[-196.71 ; 103.15]$} & 0.539 \\
\hline [1.3-1.5] & -15.70 & {$[-71.41 ; 40.01]$} & 0.579 & 12.16 & {$[-13 ; 37.33]$} & 0.342 & -121.65 & {$[-297.02 ; 53.72]$} & 0.173 \\
\hline Intercept & 590.55 & {$[550.21 ; 630.89]$} & 0.000 & 259.96 & {$[241.74 ; 278.18]$} & 0.000 & 1447.01 & {$[1320.02 ; 1574.01]$} & 0.000 \\
\hline
\end{tabular}

sulfated metanephrines, during hydrolysis of native or spiked urines indicating that $20 \mathrm{~min}$ is sufficient for hydrolysis completion (Tables 1 and 2). We also found an underestimation of the three metanephrines and an interaction between spike and $\mathrm{pH}$ for normetanephrine and metanephrine (Tables 1, 2 and 3). Therefore, the spiked and unspiked (native) urines were analyzed separately.

\subsubsection{Native urines}

Multiple linear regression analyses were carried out for native urines hydrolyzed at $\mathrm{pH} 0.9-1.1$ for $30 \mathrm{~min}$; these yielded $585 \mathrm{nmol} / \mathrm{l}$ free normetanephrine and significantly $60 \mathrm{nmol} / \mathrm{l}$ less at $\mathrm{pH} 0.5-0.7(\mathrm{p}=0.014)$ but marginally significantly $35 \mathrm{nmol} / \mathrm{l}$ more at $\mathrm{pH} 0.7-0.9(\mathrm{p}=0.095)$. S-MN at a concentration of $50 \mathrm{nmol} / \mathrm{l}$ was significantly less hydrolyzed at $\mathrm{pH}$ 0.5-0.7 than in the $\mathrm{pH}$ interval of $0.7-1.5$ (209 vs $259 \mathrm{nmol} / \mathrm{l}, \mathrm{p}<0.001$ ). The best yield for the hydrolysis of S-MT was observed at $\mathrm{pH}$ 0.7-0.9 compared to $\mathrm{pH}$ 0.9-1.1 (1599 vs $1430 \mathrm{nmol} / \mathrm{l}, \mathrm{p}<0.01$ ) (Table 1 ).The variances of the means showed that compared to $\mathrm{pH}$ 0.9-1.1, the lowest variability of hydrolysis for the highest mean concentration was observed for S-NMN at pH 0.7-0.9 (108 vs $150 \mathrm{nmol} / \mathrm{l}, \mathrm{p}=0.009$ ), for S-MN at $\mathrm{pH} 0.7-1.3$ ( 40 vs $69 \mathrm{nmol} / \mathrm{l}, \mathrm{p}=0.001$ ) and for S-MT at $\mathrm{pH}$ 0.7-0.9 (357 vs $460 \mathrm{nmol} / \mathrm{l}, \mathrm{p}=0.037$ ) (Table 3).

\subsubsection{Spiked urines}

The best hydrolysis yields and lowest variances were observed between $\mathrm{pH}$ 0.7-1.3 for exogeneous S-NMN, at pH 1.1-1.3 for S-MN and at pH 0.7-0.9 for S-MT (Tables 2 and 3).

\section{Discussion}

Our study confirmed that most metanephrines in urine controls and calibrators represent free rather than sulfoconjugates, as previously reported [18]. Therefore, commercial and in-house calibrators based on free forms are inadequate for standardization of sulfated metanephrine hydrolysis and should be replaced by "true calibrators" that correctly mimic endogenous metabolites as they complete sample preparation prior to analytical quantification.

We found that in the investigated time frame, $20 \mathrm{~min}$ is sufficient to achieve a complete hydrolysis of sulfated metanephrines without degradation of free metanephrines. For native urines, a pH between 0.7 and 0.9 seems optimal for all metanephrines whereas below or above this $\mathrm{pH}$ interval the hydrolysis yields decrease rapidly and interassay variability increases. The advantage of standardized conditions for hydrolysis is a narrowing of the reference intervals between all laboratories for sulfated metanephrines in a given population. Therefore, external quality assessment for urinary metanephrine monitoring should reflect the sole analytical variability caused by instrumental set-up with no consideration for sample preparation. Another improvement for proficiency testing would also be expected by using a synthetic source of sulfated metanephrines as calibrators. Indeed, the synthetic and endogenous S-NMN and S-MT exhibit similar behavior with an optimal $\mathrm{pH}$ hydrolysis between 0.7 and 0.9. A slight difference is observed for S-MN where the relatively large tolerance for $\mathrm{pH}$ conditions in native urines $(\mathrm{pH} 0.7-1.5)$ is restricted to $\mathrm{pH} 1.1-$ 1.3 with synthetic compounds. This discrepancy is likely due to the relatively low concentration of sulfate metanephrine in natural urine compared with the high concentration in the spiked samples (270 vs $700 \mathrm{nmol} / \mathrm{l})$, a difference which increases the variance of the assay (Tables 2 and 3).

In conclusion, the efficient and selective chemical protocols developed for the synthesis of sulfated metanephrines represent an important progress which overcomes entrenched major shortcomings in the monitoring and diagnosis of catecholamine-producing tumors. Moreover, these chemically pure compounds represent a source of calibrators and quality controls to build an effective testing needed to provide accuracy between all laboratories involved in the biochemical diagnosis of pheochromocytoma.

Table 2

Simultaneous effects of $\mathrm{pH}$ and duration on the percentage of hydrolysis of sulfated metanephrines for spiked urines.

\begin{tabular}{|c|c|c|c|c|c|c|c|c|c|}
\hline & \multicolumn{3}{|l|}{ NMN } & \multicolumn{3}{|l|}{$\mathrm{MN}$} & \multicolumn{3}{|l|}{ MT } \\
\hline & Beta & Confidence interval at $95 \%$ & p-Value & Beta & Confidence interval at $95 \%$ & p-Value & Beta & Confidence interval at 95\% & p-Value \\
\hline \multicolumn{10}{|c|}{ Time reference: $30 \mathrm{~min}$} \\
\hline $20 \mathrm{~min}$ & -16.59 & {$[-73.41 ; 40.24]$} & 0.566 & -9.70 & {$[-42.56 ; 23.16]$} & 0.561 & -78.67 & {$[-236.75 ; 79.41]$} & 0.328 \\
\hline $25 \mathrm{~min}$ & -29.40 & {$[-86.99 ; 28.19]$} & 0.315 & -5.46 & {$[-38.76 ; 27.84]$} & 0.747 & -55.90 & {$[-216.1 ; 104.31]$} & 0.492 \\
\hline $5 \mathrm{~min}$ & 15.30 & {$[-42.08 ; 72.68]$} & 0.600 & 3.86 & {$[-29.32 ; 37.04]$} & 0.819 & 13.88 & {$[-145.75 ; 173.5]$} & 0.864 \\
\hline \multicolumn{10}{|c|}{ pH reference: [0.9-1.1] } \\
\hline$[0.5-0.7]$ & -147.75 & {$[-207.93 ;-87.56]$} & 0.000 & -144.06 & {$[-178.86 ;-109.26]$} & 0.000 & -44.90 & {$[-212.33 ; 122.54]$} & 0.598 \\
\hline$[0.7-0.9]$ & -11.52 & {$[-66.81 ; 43.77]$} & 0.682 & -53.67 & {$[-85.64 ;-21.7]$} & 0.001 & 195.15 & {$[41.34 ; 348.96]$} & 0.013 \\
\hline [1.1-1.3] & 29.76 & {$[-29.03 ; 88.56]$} & 0.319 & 50.29 & {$[16.29 ; 84.29]$} & 0.004 & -65.64 & {$[-229.21 ; 97.92]$} & 0.430 \\
\hline$[1.3-1.5]$ & -157.26 & {$[-237.27 ;-77.25]$} & 0.000 & 20.79 & {$[-25.48 ; 67.05]$} & 0.377 & -330.65 & {$[-553.24 ;-108.07]$} & 0.004 \\
\hline Intercept & 448.87 & {$[398.59 ; 499.16]$} & 0.000 & 154.35 & {$[125.27 ; 183.43]$} & 0.000 & 1637.23 & {$[1497.33 ; 1777.13]$} & 0.000 \\
\hline
\end{tabular}


Table 3

Significance of mean differences from multiple regression analyses with $\mathrm{pH}$ as factors.

\begin{tabular}{|c|c|c|c|c|c|c|c|c|c|c|c|c|c|}
\hline \multicolumn{6}{|l|}{ NMN } & \multicolumn{4}{|l|}{ MN } & \multicolumn{4}{|l|}{ MT } \\
\hline $\mathrm{pH}$ & & Natural urines & p-Value & Spiked urines & p-Value & Natural urines & p-Value & Spiked urines & p-Value & Natural urines & p-Value & Spiked urines & p-Value \\
\hline \multirow[t]{2}{*}[0.5-0.7]{} & Mean & 525.91 & 0.014 & 293.49 & $<0.001$ & 209.08 & $<0.001$ & 7.57 & $<0.001$ & 1368.86 & 0.413 & 1562.65 & 0.598 \\
\hline & sd & 86.35 & $<0.001$ & 128.01 & 0.813 & 32.34 & $<0.001$ & 52.60 & 0.009 & 286.44 & 0.001 & 383.40 & 0.030 \\
\hline \multirow{2}{*}[0.7-0.9]{} & Mean & 620.51 & 0.095 & 429.46 & 0.682 & 257.00 & 0.776 & 97.53 & 0.001 & 1599.65 & 0.010 & 1799.63 & 0.013 \\
\hline & sd & 108.39 & 0.009 & 157.64 & 0.335 & 48.11 & 0.008 & 95.74 & 0.204 & 357.40 & 0.037 & 386.06 & 0.278 \\
\hline \multirow[t]{2}{*}{ [0.9-1.1] } & Mean & 585.69 & Ref & 441.45 & Ref & 259.82 & Ref & 151.62 & Ref & 1430.06 & Ref & 1608.03 & Ref \\
\hline & $\mathrm{sd}$ & 150.91 & Ref & 137.86 & Ref & 69.58 & Ref & 79.27 & Ref & 460.17 & Ref & 465.81 & Ref \\
\hline \multirow[t]{2}{*}{ [1.1-1.3] } & Mean & 592.91 & 0.750 & 471.53 & 0.319 & 270.20 & 0.333 & 201.98 & 0.004 & 1381.49 & 0.539 & 1542.49 & 0.430 \\
\hline & sd & 86.24 & $<0.001$ & 137.16 & 0.990 & 40.44 & 0.001 & 88.12 & 0.894 & 258.53 & $<0.001$ & 354.15 & 0.027 \\
\hline \multirow[t]{2}{*}{ [1.3-1.5] } & Mean & 571.18 & 0.579 & 282.52 & $<0.001$ & 272.38 & 0.342 & 171.68 & 0.377 & 1312.21 & 0.173 & 1272.74 & 0.004 \\
\hline & sd & 93.75 & 0.016 & 209.95 & 0.116 & 45.85 & 0.026 & 118.48 & 0.163 & 298.48 & 0.029 & 452.16 & 0.901 \\
\hline
\end{tabular}

\section{Acknowledgments}

The Swiss National Science Foundation and the EPFL are gratefully acknowledged for financial support (SGL). We thank Mr. Martial Rey (NMR spectrometry service, ISIC, EPFL), Dr. Laure Menin and Mr. Francisco Sepulveda (MS service, ISIC, EPFL), Mrs Marielle Dunand (Division de Pharmacologie et Toxicologie Cliniques, CHUV) for technical help and Dr. B. Testa for the careful reading of the manuscript. We are grateful to RECIPE for providing quality controls and solid-phase extraction columns.

\section{Appendix A. Supplementary data}

Supplementary data to this article can be found online at doi:10. 1016/j.cca.2012.02.023.

\section{References}

[1] Goldstein DS, Eisenhofer G, Kopin IJ. Sources and significance of plasma levels of catechols and their metabolites in humans. J Pharmacol Exp Ther 2003;305:800-11.

[2] Falany CN. Enzymology of human cytosolic sulfotransferases. FASEB J 1997;11: 206-16.

[3] Cappiello M, Giuliani L, Pacifici GM. Differential distribution of phenol and catechol sulphotransferases in human liver and intestinal mucosa. Pharmacology 1990;40:69-76.

[4] Grouzmann E, Drouard-Troalen L, Baudin E. Diagnostic accuracy of free and total metanephrines in plasma and fractionated metanephrines in urine of patients with pheochromocytoma. Eur J Endocrinol 2010;162:951-60 29.

[5] Labrosse EH, Mann JD. Presence of metanephrine and normetanephrine in normal human urine. Nature 1960;185:40.

[6] Buu NT, Angers M, Chevalier D, Kuchel O. A new method for the simultaneous analysis of free and sulfoconjugated normetanephrine, metanephrine, and 3methoxytyramine in human urine by HPLC with electrochemical detection. J Lab Clin Med 1984;104:425-32.

[7] Radjaipour M, Raster H, Liebich HM. Quantification of urinary catecholamines, their abundant metabolites, and 5-hydroxyindoleacetic acid by high performance liquid chromatography and electrochemical detection, using a single mobile phase and uniform isocratic conditions. Eur J Clin Chem Clin Biochem 1994;32:609-13.

[8] Volin P. Determination of urinary normetanephrine, metanephrine and 3methoxytyramine by high-performance liquid chromatography with electrochemical detection: comparison between automated column-switching and manual dual-column sample purification methods. J Chromatogr 1992;578:165-74.

[9] Revol A, Comoy E, Forzy G, et al. Recommended methods for the determination of catecholamines and their metabolites in urine. Significance of results in the diagnosis and follow-up of pheochromocytoma and neuroblastoma. Ann Biol Clin (Paris) 1994;52:625-37.

[10] Westerink BH, ten Kate N. 24 h excretion patterns of free, conjugated and methylated catecholamines in man. J Clin Chem Clin Biochem 1986;24:513-9.
[11] Jackman GP. A simple method for the assay of urinary metanephrines using high performance liquid chromatography with fluorescence detection. Clin Chim Acta 1982;120:137-42.

[12] Gupta RN. Improved sample preparation in determination of urinary metanephrines by liquid chromatography with electrochemical detection. Clin Chem 1990;36:538-40.

[13] Jouve J, Mariotte N, Sureau C, Muh JP. High-performance liquid chromatography with electrochemical detection for the simultaneous determination of the methoxylated amines, normetanephrine, metanephrine and 3-methoxytyramine, in urine. J Chromatogr 1983;274:53-62.

[14] Robertson D, Heath EC, Falkner FC, Hill RE, Brilis GM, Watson JT. A selective and sensitive assay for urinary metanephrine and normetanephrine using gas chromatography mass spectrometry with selected ion monitoring. Biomed Mass Spectrom 1978:5:704-8.

[15] Bertani-Dziedzic LM, Krstulovic AM, Dziedzic SW, Gitlow SE, Cerqueira S. Analysis of urinary metanephrines by reversed-phase high-performance liquid chromatography and electrochemical detection. Clin Chim Acta 1981;110:1-8.

[16] Pisano JJ. A simple analysis for normetanephrine and metanephrine in urine. Clin Chim Acta 1960;5:406-14.

[17] Fendley TW, Frings CS. A modified procedure for determining metanephrines in urine. Clin Biochem 1976;9:106-7.

[18] Singh RJ, Grebe SK, Yue B, et al. Precisely wrong? Urinary fractionated metanephrines and peer-based laboratory proficiency testing. Clin Chem 2005;51:472-3.

[19] Hegedüs B. Syntheses of sulfuric acid esters of dopamine and related compounds. Helv Chim Acta 1963;46:2604-12.

[20] Krylov VB, Ustyuzhaninaa NE, Gracheva AA, Nifantiev NE. Efficient acid-promoted per-O-sulfation of organic polyols. Tetrahedron Lett 2008;49:5877-9.

[21] El-Hiti GA. Recent advances in the synthesis of sulfonic acids. Sulfur Rep 2001;22: 217-50.

[22] Ansink HRW, Cerfontain H. Aromatic sulfonation. 114. Sulfonation of anisole, phenol, toluene and related alkyl and alkoxy derivatives with sulfur trioxide. The influence of the solvent system on the reactivity and the sulfonic acid product distribution. Recl Trav Chim Pays-Bas 1992;111:183-7.

[23] Ansink HRW, Cerfontain H. Aromatic sulfonation. 113. Reactions of sulfur trioxide with benzene derivatives containing a deactivating oxy substituent. Recl Trav Chim Pays-Bas 1992;111:215-21.

[24] Mol JA, Visser TJ. Synthesis and some properties of sulfate esters and sulfamates of iodothyronines. Endocrinology 1985;117:1-7.

[25] Hedayatullah M, Leveque JC, Denivelle L. Aryl and alkyl chlorosulfates and neutral sulfates. Action of sulfuryl chloride on alcohols. C R Acad Sci Paris Serie C 1972;274:1937-40.

[26] Hedayatullah M, Leveque JC, Denivelle L. Action of sulfuryl chloride on some phenols and alcohols. Chlorosulfates of alkyl- and arylphenols. C R Acad Sci Paris Serie C 1971;273:1444-7.

[27] Simpson LS, Widlanski TS. A comprehensive approach to the synthesis of sulfate esters. J Am Chem Soc 2006;128:1605-10.

[28] Lu CP, Ren CT, Wu SH, Chu CY, Lo LC. Development of an activity-based probe for steroid sulfatases. Chembiochem 2007;8:2187-90.

[29] Liu Y, Lien IF, Ruttgaizer S, Dove P, Taylor SD. Synthesis and protection of aryl sulfates using the 2,2,2-trichloroethyl moiety. Org Lett 2004;6:209-12.

[30] Jiang ZY, Wang YG. A mild, efficient and selective deprotection of tbutyldimethylsilyl-protected phenols using cesium carbonate. Tetrahedron Lett 2003;44:3859-61. 